\title{
Multicenter prospective evaluation of the express view reading mode for small-bowel capsule endoscopy studies
}

\section{(ㄷ)(1) $\odot$}

\author{
Authors \\ Institutions \\ 1 Gastroenterology Department, E. Herriot Hospital, Lyon, \\ France \\ 2 Gastroenterology Department, Nîmes, France \\ 3 Gastroenterology Department, Marseille, France \\ 4 Gastroenterology Department, Brest, France \\ 5 Gastroenterology Department, Nancy, France \\ 6 Gastroenterology Department, Cannes, France \\ 7 Gastroenterology Department, Avignon, France \\ 8 Gastroenterology Department, Hôpital Européen \\ Georges Pompidou, Assistance Publique Hôpitaux de \\ Paris, Université Paris Descartes, Paris, France
}

Jean-Christophe Saurin ${ }^{1}$, Philippe Jacob ${ }^{2}$, Laurent Heyries ${ }^{3}$, Christian Pesanti ${ }^{3}$, Franck Cholet ${ }^{4}$, Isaac Fassler ${ }^{5}$, James Boulant $^{6}$, Slim Bramli ${ }^{7}$, Antoin De Leusse ${ }^{1}$, Gabriel Rahmi ${ }^{8}$, and the French Society of Digestive Endoscopy (SFED)

submitted 17.10.2017

accepted after revision 2.2.2018

\author{
Bibliography \\ DOI https://doi.org/10.1055/a-0587-4788 | \\ Endoscopy International Open 2018; 06: E616-E621 \\ (c) Georg Thieme Verlag KG Stuttgart · New York \\ ISSN 2364-3722
}

\author{
Corresponding author \\ Jean-Christophe Saurin, Gastroenterology Department, \\ Hôpital E. Herriot, 5 Place d'Arsonval, 69437 Lyon Cedex 03, \\ France \\ Fax: +33-4-78861063 \\ jean-christophe.saurin@chu-lyon.fr
}

\section{ABSTRACT}

Background Reducing the reading time of capsule endoscopy films is of high priority for gastroenterologists. We report a prospective multicenter evaluation of an "express view” reading mode (Intromedic capsule system).

Methods Eighty-three patients with obscure gastrointestinal bleeding were prospectively included in 10 centers. All patients underwent small-bowel capsule endoscopy (Intromedic, Seoul, Republic of Korea). Films were read in standard mode, then a second reading was performed in express view mode at a second center. For each lesion, the precise location, nature, and relevance were collected. A consensus reading and review were done by three experts, and considered to be the gold standard.

Results The mean reading time of capsule films was 39.7 minutes (11-180 minutes) and 19.7 minutes (440 minutes) by standard and express view mode, respectively $\left(P<1 \times 10^{-4}\right)$. The consensus review identified a significant lesion in $44 / 83$ patients $(53.0 \%)$. Standard reading and express view reading had a $93.3 \%$ and $82.2 \%$ sensitivity, respectively (NS). Consensus review identified 70 significant images from which standard reading and express view reading detected $58(82.9 \%)$ and $55(78.6 \%)$, respectively. The informatics algorithm detected 66/70 images (94.3\%) thus missing four small-bowel angiodysplasia.

Conclusion The express view algorithm allows an important shortening of Intromedic capsule film reading time with a high sensitivity.

\section{Introduction}

Capsule endoscopy is the first choice small-bowel examination for most gastroenterologists worldwide in patients presenting with obscure (overt or occult) gastrointestinal bleeding (OGIB), because of simple use, low risk, and high sensitivity compared to all other small-bowel imaging modalities [1,2]. Capsule endoscopy is a very sensitive examination for the detection of small-bowel lesions, as assessed by long-term follow-up of patients after negative capsule endoscopy: only $10 \%$ of patients present with ongoing bleeding related to a missed small-bowel lesion [3]. The main limitation of capsule endos- copy is the substantial time needed for capsule reading by highly qualified medical personnel. Most series [4-6], report a mean reading time of 50 to 60 minutes. In addition, the focus and attention to detail that are necessary to efficiently read a film are difficult to maintain during the whole reading $[4,7]$. For this reason, attempts have been made to transfer capsule reading to nurses or other similarly qualified personnel, with relatively satisfying results $[8,9]$. Informatics approaches to solving the reading time dilemma have also been proposed, with variable results. This approach needs clinical evaluation and validation $[10,11]$. 
Nowadays, very few informatics algorithms for accelerated reading have been validated, although almost all capsule systems propose such informatics tools. This includes the SBI (small-bowel blood indicator, Given Imaging/Medtronic), that proved to be of poor sensitivity and specificity $[12,13]$, and the Quick-view algorithm that was validated through a retrospective blinded double-reading trial published in 2012 [14]. Since that study, very few evaluations of informatics reading algorithms have been published, and no prospective evaluation [15]. Developing such complex algorithms requires specifically large image databases that have been produced through international collaborations [16]. The Intromedic company recently proposed a new informatics algorithm named "express view" to reduce the reading time of small-bowel capsule films by skipping repetitive and non-significant small-bowel images on the film. To the best of our knowledge, this algorithm has never been evaluated. We thus performed a prospective, multicenter evaluation of the express view algorithm in unselected films from 10 French gastroenterology centers, with the aim of evaluating the sensitivity and specificity of an express view reading compared to the previous standard reading of the capsule film.

\section{Materials and methods}

\section{Small-bowel capsule endoscopy and population}

The study includes 83 small-bowel capsule films from 83 consecutive unselected patients included prospectively in one of 10 experienced French centers belonging to the French Society of Digestive Endoscopy (SFED). The indication for capsule endoscopy was obscure (overt or occult) bleeding in all cases, with negative bidirectional endoscopy in all cases. The capsule endoscopy (Mirocam, Intromedic, Seoul, Republic of Korea) procedure was performed in each center following the usual local procedures including preparation and prokinetics in some centers: as the main objective of the study was the reading time, and as each capsule film was read in both standard mode and express view mode, being its own control, differences in preparation quality or small-bowel transit time could not modify the results. Characteristics of the Intromedic capsule system are: a resolution of $320 \times 320$, a field of view of $170^{\circ}$ and a frame rate of 3 images per second. Each capsule film was read initially by one experienced local reader (standard or initial reading). Information on patient characteristics, indication for capsule, findings according to the SFED classification of relevance [17], and reading time, were recorded prospectively. All observed lesions were described in the reports in terms of nature, location, and relevance. All 83 films were registered on the same hard disk, and then dispatched to the different centers for express view analysis.

\section{Express view reading}

For each center, one reader was in charge of capsule video analysis in express view mode, and was blinded to any information about previous diagnosis. This reader was thus in charge of the analysis of films from one other center. The readers were asked to read only in express view mode and to select all pathological images, identify their relevance, and define a final diagnosis

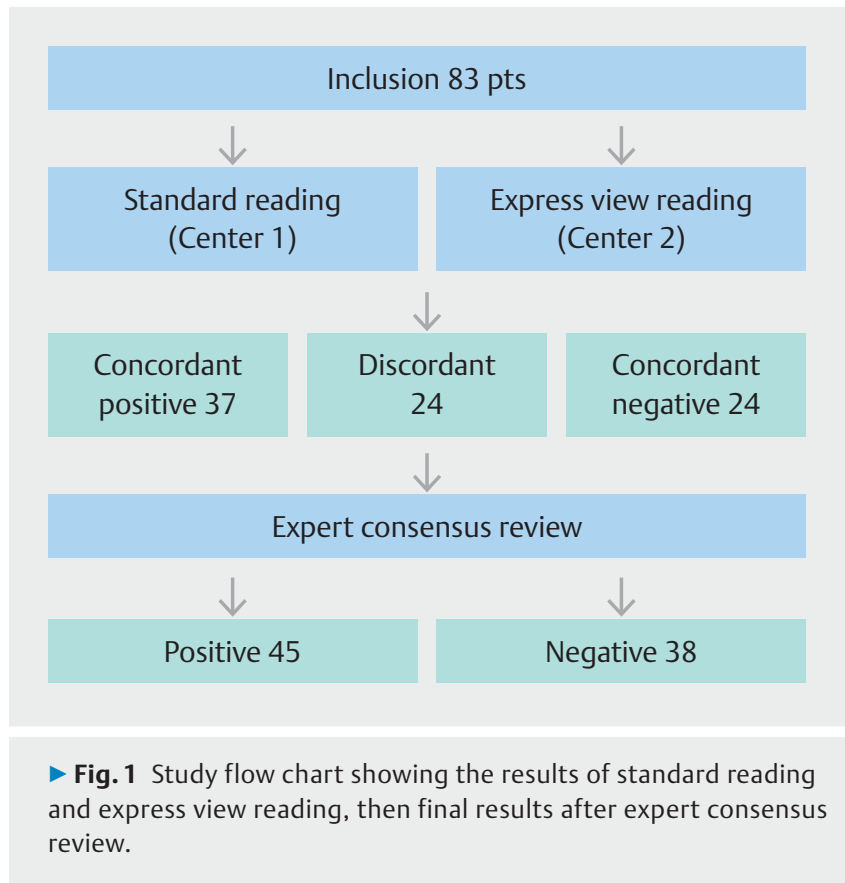

corresponding to the most significant image in the entire (including stomach and colon) capsule film. Readers were allowed to choose their preferred image reading frame. The results of the express view reading and of the initial reading were saved on a pro forma.

\section{Interpretation of the results (see flow chart, > Fig.1)}

A comparison was made for each case between the results of the initial reading and that of the express view reading. In cases with concordant (positive or negative) diagnosis between the standard and express view reading, no complementary reading was performed. In case of a discordant final diagnosis and/or different lesions observed, a secondary expert review was performed by three readers (JCS, PJ, GR), all three having experience of more than 500 capsule readings, and a consensus decision was made. The result of this consensus review was considered to be the gold standard result and compared with the results of the standard reading and of the express view reading. We performed a per patient analysis based on the main diagnosis obtained by capsule endoscopy for each patient (i.e. smallbowel tumor, angiodysplasia, ulceration, etc.). We performed a per lesion analysis by including all significant (P1 or P2) lesions according to the SFED classification [17].

\section{Statistical analysis}

Sensitivity values were presented as a percentage (95\% confidence interval). McNemar's test was used to compare the express view and standard reading sensitivity and specificity results using the expert review as a gold standard. We used SPSS 12.0 version software (SPSS Inc, Chicago, IL, United States) in a Windows XP (Microsoft, Seattle, WA, United States) environment. A $P$ value $<0.05$ was considered to be statistically significant. 


\section{Results}

Between February 2013 and February 2016, 83 patients with obscure/overt digestive bleeding were included (43 women, mean age 62.5 years, range $21-85$ ). The 83 CE films were read in express view mode without technical issue, with a mean reading time of 18.3 minutes (range 3-41 minutes) versus 37.7 minutes (range $11-180$ minutes, $P<0.0001$, Student's bilateral $t$ test) for standard reading mode. Evaluation of landmarks in normal and express view reading modes yielded comparable mean gastric and small-bowel transit times: 50.1 and 223.1 minutes (normal mode) and 49.6 and 210.4 minutes (express view mode). The algorithm selection allowed a mean reduction in the number of images from a mean of 80596 images (range 123323-52248) to a mean of 22087 (5196-43017) thus resulting in a mean $70 \%$ reduction in the number of images in one complete capsule film.

\section{Per patient analysis}

From the 83 patients prospectively included, 45 (54.2\%) had a main diagnosis after consensus review of the whole series ( $\vee$ Fig.1). The final diagnosis was angiodysplasia in 29 cases, erosion-ulcerations in 10 cases, ongoing bleeding without evidenced diagnosis in four cases and tumor in two cases. Stand- ard reading identified 42 (sensitivity $93.3 \%$ ) of these main diagnoses and express view reading identified 37 (82.2\%) of these diagnoses ( $\triangleright$ Table 1 ). Standard reading missed one P2 gastric tumor, one P2 cecal ongoing bleeding, and one P1 gastritis. Express view reading missed four cases of small-bowel angiodysplasia (including two cases where the image was skipped by the express view algorithm, \Fig.2), 1 jejunal ulcer (\Fig. 3 EV1), one $\mathrm{P} 2$ duodenal ulcer, and 2 gastric lesions (P1 gastritis and P2 ulcer). Thus, six out of eight main diagnoses missed at express view reading were present on the express view film but missed by the readers, and two were missed because of the informatics selection.

\section{Per lesion analysis}

Seventy significant (P1 or P2) images were identified by the readers according to the expert review final evaluation. Standard reading (normal mode) identified 58 from these 70 images $(82.9 \%)$, compared to 55 images identified in express view mode $(78.6 \%, P=0.388)$. On the express view film, 66/70 images were present (sensitivity of the algorithm $94.3 \%$ ). Thus, at initial reading, readers missed 12 images present on the entire capsule films (17.1\%) and express view readers missed 11 images present on the express view-selected capsule film (16.6\%, - Fig.3). The informatics algorithm skipped four significant

\begin{tabular}{|l|l|l|l|}
\hline \multicolumn{1}{|l|}{ Table 1 Sensitivity of initial reading and express view reading (95\% confidence interval) in a per patient and per lesion analysis. } \\
\hline
\end{tabular}
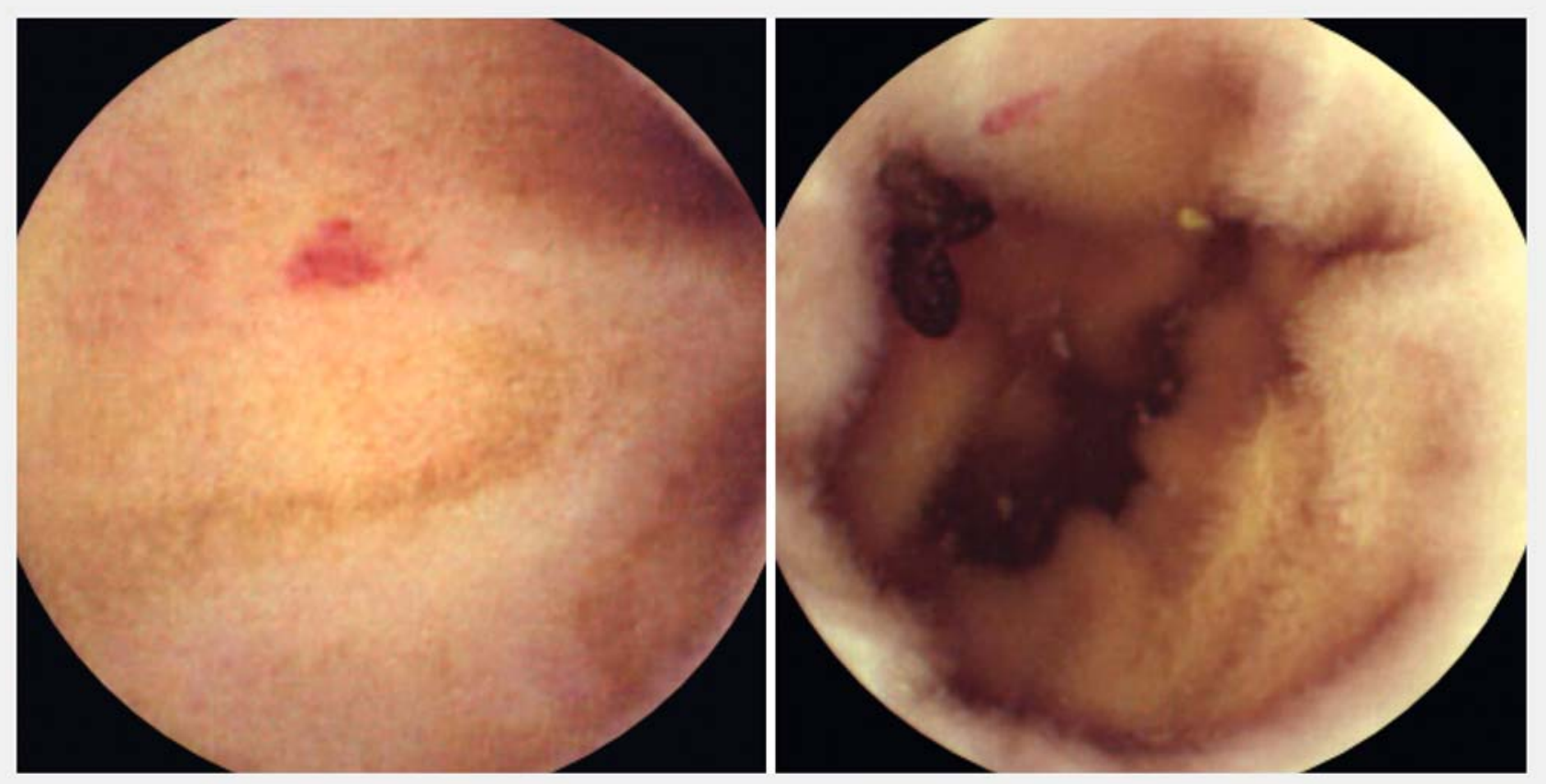

Fig. 2 Two P2 and P1 angiodysplasias skipped by the express view algorithm, thus absent from the express view film. 


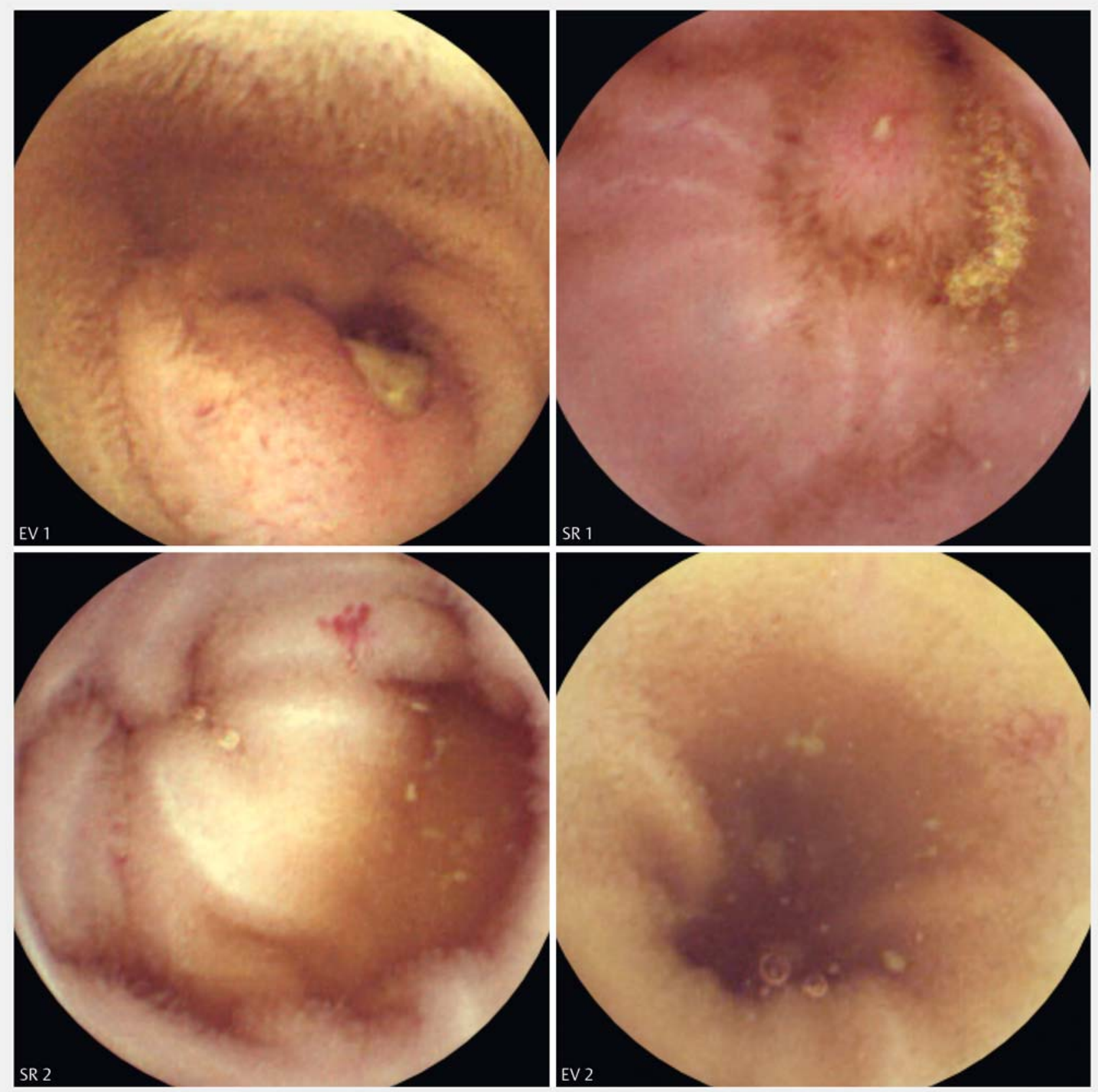

- Fig. 3 Different small-bowel diagnoses missed by the readers in express view (EV) or standard reading (SR) mode but present on the corresponding films. EV1: P2 jejunal ulceration; SR1: Aphthous P1 ulceration in the ileum; SR2: P2 angiodysplasia; EV2: P1 angiodysplasia.

images from the initial film that were all small-bowel angiodysplasia, classified as highly relevant in two cases and of uncertain/intermediate relevance in two cases. - Fig. 2 shows two angiodysplasias skipped by the informatics algorithm.

\section{Discussion}

There is a high expectation of efficient algorithms allowing reading time reduction for small-bowel capsule endoscopy films, as the mean reading time proved to be significant, from 30 to 60 minutes $[4,17,18]$. Interestingly, numerous algo- rithms exist for different capsule endoscopy devices, but very few prospective blinded evaluations have been performed at the present time. One retrospective blinded multicentric evaluation validated the quick-view algorithm of the Given Imaging (now Medtronic) small-bowel capsule endoscopy device [14]. That study showed a $93 \%$ sensitivity of the informatics algorithm for significant (P1 or P2) lesions in a setting of obscure bleeding. The present study shows that the express view informatics algorithm developed for the Intromedic device and software has a $94.2 \%$ sensitivity for the detection of significant lesions. This sensitivity for significant lesions should be the main 
objective of any prospective evaluation of these algorithms, as the major consideration from the patient's point of view is that no important diagnostic should be missed (skipped) by the selection algorithm. Thus, the express view algorithm, like the quick-view algorithm previously, seems to be a very efficient and safe selection algorithm for clinical use, although confirmative studies are needed.

The secondary end point of such reading studies is the sensitivity of the reading. It is important, however, to differentiate this sensitivity from that of the informatics algorithm, as this is "operator-dependent" and represents the efficacy of the reader rather than the efficacy of the informatics algorithm or of the device itself. In the present study, the sensitivity of reading was limited in express view mode, although not significantly different from that of the standard reading. This represents real life practice: we included readers from university hospitals, general hospitals, and private practice. Although all centers had a large experience with capsule reading, differences of reading interpretation is a characteristic of multicenter studies that we observed in numerous previous studies $[14,19,20]$. On the other hand, image selection by an informatics algorithm may reduce the number of consecutive images of an angiodysplasia, as an example, on the express view film compared to the noncontrast film, which occurred in a few cases (not shown) in this study. However, this did not explain the lower per patient sensitivity of express mode reading in this study, as, in contrast, the per lesion sensitivity of express view reading was slightly higher (but not significantly) compared to the per lesion sensitivity of normal reading. In fact, this may actually represent the real life variability of multicenter capsule reading and argues in favor of an evolution toward automatic reading and selection by computer: this is certainly part of the future of capsule endoscopy. In this setting, the main result of such a study is the theoretical sensitivity of the informatics algorithm. The use of an expert review, considered to be the final "gold standard", may be criticized as it represents some bias of expert judgment compared to the "real life" reading. However, we showed in previous studies that the subjectivity of capsule reading is a constant feature of multicenter studies, with a significant proportion of false positive/false negative images responsible for confusing results when evaluating the sensitivity of reading, and especially when evaluating an informatics algorithm for image selection $[7,14,21]$.

Is the express view algorithm now ready for clinical use? The theoretical sensitivity of more than $94 \%$ is rather reassuring, although confirmation by an independent study would be helpful. In this setting, one could use the express view mode to make a first reading, that could be considered to be sufficient in those patients with significant findings at the end of this first reading. In those patients with a negative express view reading, a reading of the "complementary film" could be done, whenever available on the Intromedic software. On the other hand, simple patient surveillance followed by a complete reading in those with recurrent bleeding is an alternative. This approach may yield precious time saving for gastroenterologists.

\section{Competing interests}

J.C. Saurin and G. Rhami are occasional consultants for Medtronic, Capsovision, and Intromedic.

References

[1] Marmo R, Rotondano G, Piscopo R et al. Meta-analysis: capsule enteroscopy vs. conventional modalities in diagnosis of small bowel diseases. . Aliment Pharmacol Ther 2005; 22: 595-604

[2] Triester SL, Leighton JA, Leontiadis Gl et al. A meta-analysis of the yield of capsule endoscopy compared to other diagnostic modalities in patients with obscure gastrointestinal bleeding. Am J Gastroenterol 2005; 100: $2407-2418$

[3] Lai LH, Wong GL, Chow DK et al. Long-term follow-up of patients with obscure gastrointestinal bleeding after negative capsule endoscopy. Am J Gastroenterol 2006; 101: 1224-1228

[4] Ell C, Remke S, May A et al. The first prospective controlled trial comparing wireless capsule endoscopy with push enteroscopy in chronic gastrointestinal bleeding. Endoscopy 2002; 34: 685-689

[5] Hartmann D, Schmidt $\mathrm{H}$, Bolz $\mathrm{G}$ et al. A prospective two-center study comparing wireless capsule endoscopy with intraoperative enteroscopy in patients with obscure GI bleeding. Gastrointest Endosc 2005; $61: 826-832$

[6] Lewis BS, Swain P. Capsule endoscopy in the evaluation of patients with suspected small intestinal bleeding: Results of a pilot study. Gastrointest Endosc 2002; 56: 349-353

[7] Saurin JC, Delvaux M, Vahedi K et al. Clinical impact of capsule endoscopy compared to push enteroscopy: 1-year follow-up study. Endoscopy 2005; 37: $318-323$

[8] Levinthal GN, Burke CA, Santisi JM. The accuracy of an endoscopy nurse in interpreting capsule endoscopy. Am J Gastroenterol 2003; 98: $2669-2671$

[9] Riphaus A, Richter S, Vonderach M et al. Capsule endoscopy interpretation by an endoscopy nurse - a comparative trial. Z Gastroenterol 2009; 47: 273-276

[10] Gan T, Wu JC, Rao NN et al. A feasibility trial of computer-aided diagnosis for enteric lesions in capsule endoscopy. World J Gastroenterol 2008; 14: 6929-6935

[11] lakovidis DK, Tsevas S, Polydorou A. Reduction of capsule endoscopy reading times by unsupervised image mining. Comput Med Imaging Graph 2009; 34: $471-478$

[12] D’Halluin PN, Delvaux M, Lapalus MG et al. Does the "Suspected Blood Indicator" improve the detection of bleeding lesions by capsule endoscopy? Gastrointest Endosc 2005; 61: 243-249

[13] Buscaglia JM, Giday SA, Kantsevoy SV et al. Performance characteristics of the suspected blood indicator feature in capsule endoscopy according to indication for study. Clin Gastroenterol Hepatol 2008; 6: $298-301$

[14] Saurin JC, Lapalus MG, Cholet F et al. Can we shorten the small-bowel capsule reading time with the "Quick-view" image detection system? Dig Liver Dis 2012; 44: 477 - 481

[15] Halling ML, Nathan T, Kjeldsen J et al. High sensitivity of quick view capsule endoscopy for detection of small bowel Crohn's disease. J Gastroenterol Hepatol 2014; 29: $992-996$

[16] Karargyris A, Koulaouzidis A. Capsule-odometer: a concept to improve accurate lesion localisation. World J Gastroenterol 2013; 19: $5943-5946$

[17] Saurin JC, Delvaux M, Gaudin JL et al. Diagnostic value of endoscopic capsule in patients with obscure digestive bleeding: blinded comparison with video push-enteroscopy. Endoscopy 2003; 35: 576-584 
[18] Costamagna G, Shah SK, Riccioni ME et al. A prospective trial comparing small bowel radiographs and video capsule endoscopy for suspected small bowel disease. Gastroenterology 2002; 123: 999 1005

[19] Pioche M, Filoche B, Jacob P et al. Randomized prospective comparison of the diagnostic yield of PillCam SB $2^{\circledR}$ and Mirocam ${ }^{\circledR}$ videocapsules in patients with obscure digestive bleeding. Gastrointest Endosc 2011; 73: $1181-1188$
[20] Pioche M, Vanbiervliet G, Jacob P et al. Prospective randomized comparison between axial- and lateral-viewing capsule endoscopy systems in patients with obscure digestive bleeding. Endoscopy 2013; 46: $479-484$

[21] Delvaux M, Fassler I, Gay G. Clinical usefulness of the endoscopic video capsule as the initial intestinal investigation in patients with obscure digestive bleeding: validation of a diagnostic strategy based on the patient outcome after 12 months. Endoscopy 2004; 36: 1067 1073 